\title{
L-Fuzzy Vector Subspaces and Its Fuzzy Dimension
}

\author{
Chun'e Huang1*, Yan Song², Xiruo Wang² \\ ${ }^{1}$ College of Biochemical Engineering, Beijing Union University, Beijing, China \\ ${ }^{2}$ Mudanjiang Normal University, Mudanjiang, China \\ Email: ^hce_137@163.com, ^hchune@buu.edu.cn, 46470102@126.com,13836309352@126.com
}

How to cite this paper: Huang, C.E, Song, Y. and Wang, X.R. (2016) L-Fuzzy Vector Subspaces and Its Fuzzy Dimension. Advances in Linear Algebra \& Matrix Theory, 6, 158-168

http://dx.doi.org/10.4236/alamt.2016.64015

Received: November 8, 2016

Accepted: December 25, 2016

Published: December 28, 2016

Copyright $\odot 2016$ by authors and Scientific Research Publishing Inc. This work is licensed under the Creative Commons Attribution International License (CC BY 4.0).

http://creativecommons.org/licenses/by/4.0/

\section{Abstract}

In this paper, we introduce the definition of $L$-fuzzy vector subspace, define its dimension by an $L$-fuzzy natural number. For a finite-dimensional $L$-fuzzy vector subspace, we prove that the equality $\operatorname{dim}\left(\tilde{E}_{1}+\tilde{E}_{2}\right)+\operatorname{dim}\left(\tilde{E}_{1} \cap \tilde{E}_{2}\right)=\operatorname{dim} \tilde{E}_{1}+\operatorname{dim} \tilde{E}_{2}$ holds without any restricted conditions. At the same time, we deduce that the formula $\operatorname{dim}(\widetilde{\operatorname{im}} f)+\operatorname{dim}(\widetilde{\operatorname{kef}} f)=\operatorname{dim} \tilde{E}$ holds.

\section{Keywords}

$L$-Fuzzy Sets, $L$-Fuzzy Vector Subspace, $L$-Fuzzy Dimension

\section{Introduction}

Firstly, fuzzy vector subspace was introduced by Katsaras and Liu [1]. Then its properties and characters were investigated (see [2] [3] [4] [5], etc). The dimension of a fuzzy vector space was defined as a $n$-tuple by Lowen [6]. Subsequently, it was defined as a non-negative real number or infinity by Lubczonok [5], and proved that the formula

$$
\operatorname{dim}\left(\tilde{E}_{1}+\tilde{E}_{2}\right)+\operatorname{dim}\left(\tilde{E}_{1} \cap \tilde{E}_{2}\right)=\operatorname{dim} \tilde{E}_{1}+\operatorname{dim} \tilde{E}_{2}
$$

is valid under certain conditions, where $\tilde{E}_{1}$ and $\tilde{E}_{2}$ are fuzzy vector spaces. Recently, basis and dimension of a fuzzy vector space were redefined as a fuzzy set and a fuzzy natural number by Shi and Huang [7], respectively. Under the definitions, more properties of (crisp) vector spaces were correct in fuzzy vector spaces.

In this paper, we generalize the results in [7] to $L$ lattice, and prove that some formulas still hold in the lattice $L$. In particular, we present the definition of $L$-fuzzy vector subspace and its -fuzzy dimension. The $L$-fuzzy dimension of a finite dimensional fuzzy vector subspace is a fuzzy natural number. We prove that (1) holds without any re- 
stricted conditions and $\operatorname{dim}(\widetilde{\operatorname{kef}} f)+\operatorname{dim}(\widetilde{\operatorname{im}} f)=\operatorname{dim} \tilde{E}$ holds.

\section{Preliminaries}

Given a set $X$ and a completely distributive lattice $L$, we denote the power set of $X$ and the set of all $L$-fuzzy sets on $X$ (or $L$-sets for short) by $2^{X}$ and $L^{X}$, respectively. For any $A \subseteq X$, we denote the cardinality of $A$ by $|A|$.

An element $a$ in $L$ is called a prime element if $a \geq b \wedge c$ implies $a \geq b$ or $a \geq c$. $a$ in $L$ is called co-prime if $a \leq b \vee c$ implies $a \leq b$ or $a \leq c$ [8]. The set of nonunit prime elements in $L$ is denoted by $P(L)$. The set of non-zero co-prime elements in $L$ is denoted by $J(L)$.

The binary relation $<$ in $L$ is defined as follows: for $a, b \in L, a<b$ if and only if for every subset $D \subseteq L$, the relation $b \leq \sup D$ always implies the existence of $d \in D$ with $a \leq d$ [9]. $\{a \in L: a<b\}$ is called the greatest minimal family of $b$ in the sense of [10], denoted by $\beta(b)$, and $\beta^{*}(b)=\beta(b) \cap J(L)$. Moreover, for $b \in L$, we define $\alpha(b)=\left\{a \in L: a<{ }^{o p} b\right\}$ and $\alpha^{*}(b)=\alpha(b) \cap P(L)$. In a completely distributive lattice $L$, there exist $\alpha(b)$ and $\beta(b)$ for each $b \in L$, and $b=\vee \beta(b)=\wedge \alpha(b)$ (see [10]).

In [10], Wang thought that $\beta(0)=\{0\}$ and $\alpha(1)=\{1\}$. In fact, it should be that $\beta(0)=\varnothing$ and $\alpha(1)=\varnothing$.

Throughout this paper, $L$ denotes a completely distributive lattice, and $E$ is a crisp vector space. We often do not distinguish a crisp subset $A$ of $E$ and its characteristic function $\chi_{A}$.

If $A \in L^{X}$ and $a \in L$, we can define

$$
\begin{gathered}
A_{[a]}=\{x \in X: A(x) \geq a\}, \quad A_{(a)}=\{x \in X: a \in \beta(A(x))\} . \\
A^{[a]}=\{x \in X: a \notin \alpha(A(x))\}, A^{(a)}=\{x \in X: A(x) \not \leq a\} .
\end{gathered}
$$

Some properties of these cut sets can be found in [11]-[16].

In [17] Shi introduced the concept of $L$-fuzzy natural numbers(denoted by $\mathbb{N}(L)$ ), defined their operations and discussed the relation of $\alpha$-cut sets. We simply recall as follows: for any $\lambda, \mu \in \mathbb{N}(L), a \in L$,

(1) $(\lambda+\mu)_{(a)} \subseteq \lambda_{(a)}+\mu_{(a)} \subseteq \lambda_{[a]}+\mu_{[a]} \subseteq(\lambda+\mu)_{[a]}$;

(2) $(\lambda+\mu)^{(a)} \subseteq \lambda^{(a)}+\mu^{(a)} \subseteq \lambda^{[a]}+\mu^{[a]} \subseteq(\lambda+\mu)^{[a]}$;

(3) For any $\lambda, \mu \in \mathbb{N}(L)$ and $a \in P(L)$, it follows that $(\lambda+\mu)^{(a)}=\lambda^{(a)}+\mu^{(a)}$.

\section{L-Fuzzy Vector Subspaces}

Definition 3.1. $L$-fuzzy vector subspace is a pair $\tilde{E}=(E, \mu)$ where $E$ is a vector space on field $F, \mu: E \rightarrow L$ is a map with the property that for any $x, y \in E, k, l \in F$, we have $\mu(k x+l y) \geq \mu(x) \wedge \mu(y)$.

In this definition, when $L=[0,1], L$-fuzzy vector subspace is exactly the fuzzy vector subspace defined in [1]. We denote the family of $L$-fuzzy vector subspaces by LFVS .

Let $\tilde{E}=(E, \mu)$ be a member of LFVS, we denote 


$$
\begin{aligned}
& \tilde{E}_{[a]}=\mu_{[a]}=\{x \in E: \mu(x) \geq a\}, \quad \tilde{E}_{(a)}=\mu_{(a)}=\{x \in E: a \in \beta(\mu(x))\} . \\
& \tilde{E}^{[a]}=\mu^{[a]}=\{x \in E: a \notin \alpha(\mu(x))\}, \quad \tilde{E}^{(a)}=\mu^{(a)}=\{x \in E: \mu(x) \not a\} .
\end{aligned}
$$

We can obtain some properties of LFVS analogous to fuzzy vector subspaces as follows.

Theorem 3.2. Let $\tilde{E}=(E, \mu)$ be a member of LFVS, then

(1) $\mu(0)=\sup _{x \in E} \mu(x)$.

(2) For any $k \in F \backslash\{0\}$ and $x \in E, \mu(k x)=\mu(x)$.

The prove is trivial and omitted.

Remark: Since $L$ is a completely distributive lattice, the property that if $\mu(x) \neq \mu(y)$, then $\mu(x+y)=\mu(x) \wedge \mu(y)$ not holds for LFVS. This can be seen from the following example.

Example 3.3. Let $L$ be a completely distributive lattice with four elements as follows.

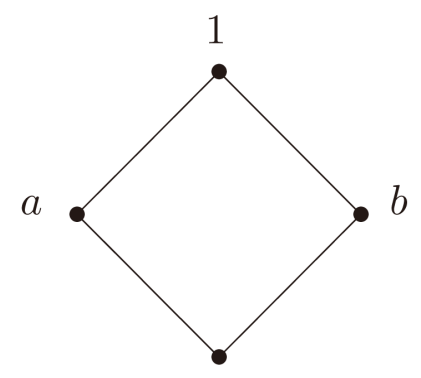

0

Let $\tilde{E}=\left(\mathbb{R}^{2}, \mu\right)$ be an $L$-fuzzy vector subspace on $\mathbb{R}^{2}$ where $\mu$ is defined by

$$
\mu(x)= \begin{cases}1, & x=(0,0) . \\ a, & x \in\{(y, 0): y \in \mathbb{R} \backslash\{0\}\} . \\ b, & x \in\{(0, y): y \in \mathbb{R} \backslash\{0\}\} . \\ 0, & \text { otherwise. }\end{cases}
$$

We can easily check $\tilde{E}$ is an $L$-fuzzy vector subspace on $\mathbb{R}^{2}$. Suppose that $x=(3,2)$ and $y=(0,-2)$, then $\mu(x+y)=\mu(3,0)=a>\mu(x) \wedge \mu(y)=0 \wedge b=0$. This example illustrates for $L$-fuzzy vector subspace $\mu(x) \neq \mu(y)$, $\mu(x+y)>\mu(x) \wedge \mu(y)$.

Theorem 3.4. Let $E$ be a vector space, $\mu \in L^{E}$ and $\tilde{E}=(E, \mu)$. Then the following statements are equivalent:

(1) $\tilde{E}$ is an $L$-fuzzy vector subspace.

(2) (a) For all $x \in E$ and $k \in F, \mu(k x) \geq \mu(x)$.

(b) For any $x, y \in E, \mu(x+y) \geq \mu(x) \wedge \mu(y)$.

(3) For any $x_{1}, \cdots, x_{r} \in E$ and $k_{1}, \cdots, k_{r} \in F$, where $r$ is a finite natural number, we have 


$$
\mu\left(\sum_{i=1}^{r} k_{i} x_{i}\right) \geq \underset{i=1}{r} \mu\left(x_{i}\right) .
$$

The prove is trivial and omitted.

In the following paper, the vector spaces we discuss are finite-dimensional. For their $L$-fuzzy vector subspaces, the following observation will be useful.

Remark: Let $\tilde{E}=(E, \mu)$ be a member of LFVS. Suppose that $\mu(E)=\{\mu(x): x \in E\}$. Since $E$ is finite-dimensional vector space, denotes $\operatorname{dim} E=n$, then $\mu(E)$ is a finite subset of $L$.

In the fact, let $B$ be a basis of $E$, then $|B|=n$. Suppose that $\mu(E)$ is infinite, then for all $a \in L$, the total number of $\tilde{E}_{[a]}$ is infinite. Since $B \cap \tilde{E}_{[a]}$ is a basis of $\tilde{E}_{[a]}$, we have $\tilde{E}_{[a]}=\left\langle B \cap \tilde{E}_{[a]}\right\rangle$. Again since $B$ is finite, the total number of $\tilde{E}_{[a]}$ is also finite. It contradicts with the hypothesis. Therefore $\mu(E)$ is a finite subset of $L$ with at most $2^{n}+1$ values; $2^{n}$ values which can be attained at the vectors of $E \backslash\{0\}$ and the maximum which is attained at 0 .

Theorem 3.5. Let $E$ be a vector space, $\mu \in L^{E}$ and $\tilde{E}=(E, \mu)$. Then the following statements equivalent:

(1) $\tilde{E}$ is an $L$-fuzzy vector subspace.

(2) For all $a \in L, \tilde{E}_{[a]}$ is a vector space.

(3) For all $a \in J(L), \tilde{E}_{[a]}$ is a vector space.

(4) For all $a \in L, \tilde{E}^{[a]}$ is a vector space.

(5) For all $a \in P(L), \tilde{E}^{[a]}$ is a vector space.

(6) For all $a \in P(L), \tilde{E}^{(a)}$ is a vector space.

Proof. We prove $(1) \Leftrightarrow(4)$ and $(1) \Leftrightarrow(6)$, the others can be proved analogously.

$(1) \Rightarrow(4)$ We show that $\tilde{E}^{[a]}$ is a vector space as follows. Suppose that $x, y \in \tilde{E}^{[a]}$, then $a \notin \alpha(\mu(x))$ and $a \notin \alpha(\mu(y))$, i.e. $a \notin \alpha(\mu(x)) \cup \alpha(\mu(y))=\alpha(\mu(x) \wedge \mu(y))$.

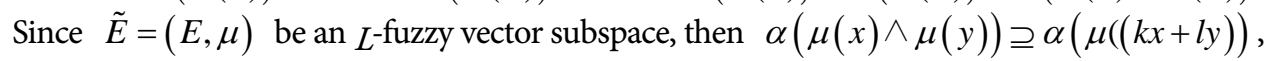
we have $a \notin \alpha(\mu(k x+l y))$, this means $k x+l y \in \tilde{E}^{[a]}$. Therefore $\tilde{E}^{[a]}$ is a vector space.

$(4) \Rightarrow(1)$ Suppose that for all $a \in L, \tilde{E}^{[a]}$ is a vector space. Let $x, y \in E$ and $k, l \in F$. Since $\tilde{E}^{[a]}$ is a vector space, then $k x+l y \in \tilde{E}^{[a]}$ if and only if $x \in \tilde{E}^{[a]}$ and $y \in \tilde{E}^{[a]}$. We have

$$
\begin{aligned}
\mu(k x+l y) & =\wedge \underset{a \in L}{\wedge}\left(a \wedge \tilde{E}^{[a]}\right)(k x+l y) \\
& =\wedge \underset{a \in L}{\wedge}\left(a \vee\left(\tilde{E}^{[a]}(x) \wedge \tilde{E}^{[a]}(y)\right)\right) \\
& =\left(\underset{a \in L}{\wedge}\left(a \vee \tilde{E}^{[a]}(x)\right)\right) \wedge\left(\widehat{a \in L}\left(a \vee \tilde{E}^{[a]}(y)\right)\right) \\
& =\mu(x) \wedge \mu(y) .
\end{aligned}
$$

Therefore $\tilde{E}$ is an $L$-fuzzy vector subspace.

$(1) \Rightarrow(6)$ Suppose that $x, y \in E^{(a)}$, then $\mu(x) \not \leq a$ and $\mu(y) \not \leq a$. Since $a \in P(L)$, then $\mu(x) \wedge \mu(y) \not \leq a$. Because $\tilde{E}=(E, \mu)$ is an $L$-fuzzy vector subspace, we can have $\mu(k x+l y) \not \leq a$, this implies $k x+l y \in E^{(a)}$. Thus $E^{(a)}$ is a vector space.

$(6) \Rightarrow(1)$ Let $x, y \in E$ and $k, l \in F$. Since $\tilde{E}^{(a)}$ is a vector space, then 
$k x+l y \in \tilde{E}^{(a)}$ if and only if $x \in \tilde{E}^{(a)}$ and $y \in \tilde{E}^{(a)}$. We have the following implications.

$$
\begin{aligned}
\mu(k x+l y) & =\underset{a \in P(L)}{\wedge}\left(a \vee \tilde{E}^{(a)}\right)(k x+l y) \\
& =\underset{a \in P(L)}{\wedge}\left(a \vee\left(\tilde{E}^{(a)}(x) \wedge \tilde{E}^{(a)}(y)\right)\right) \\
& =\left(\underset{a \in P(L)}{\wedge}\left(a \vee \tilde{E}^{(a)}(x)\right)\right) \wedge\left(\hat{A}_{a \in P(L)}\left(a \vee \tilde{E}^{(a)}(y)\right)\right) \\
& =\mu(x) \wedge \mu(y) .
\end{aligned}
$$

Therefore $\tilde{E}$ is an $L$-fuzzy vector subspace.

Theorem 3.6. Let $E$ be a vector space, $\mu: E \rightarrow L$ be a map, $\tilde{E}=(E, \mu)$, and for all $a, b \in L, \beta(a \wedge b)=\beta(a) \cap \beta(b)$. Then the following statements equivalent.

(1) $\tilde{E}$ is an $L$-fuzzy vector subspace.

(2) For all $a \in L, \tilde{E}_{(a)}$ is a vector space.

Proof. (1) $\Rightarrow(2)$ Suppose that $x, y \in \tilde{E}_{(a)}$, then $a \in \beta(\mu(x))$ and $a \in \beta(\mu(y))$, i.e. $a \in \beta(\mu((x)) \cap \beta(\mu(y))$. Since for all $a, b \in L, \beta(a \wedge b)=\beta(a) \cap \beta(b)$ and $\tilde{E}$ is an $L$-fuzzy vector subspace, we can know $a \in \beta(\mu(x) \wedge \mu(y)) \subseteq \beta(\mu(a x+b y))$, this implies $a x+b y \in \tilde{E}_{(a)}$. Therefore $\tilde{E}_{(a)}$ is a vector space.

$(2) \Rightarrow(1)$ Suppose that for all $a \in L, \tilde{E}_{(a)}$ is a vector space. Let $x, y \in E$ and $k, l \in F$. Since $\tilde{E}_{(a)}$ is a vector space, then $k x+l y \in \tilde{E}_{(a)}$ if and only if $x \in \tilde{E}_{(a)}$ and $y \in \tilde{E}_{(a)}$. We have

$$
\begin{aligned}
& \mu(k x+l y)=\underset{a \in L}{\vee}\left(a \wedge \tilde{E}_{(a)}\right)(k x+l y) \\
& =\underset{a \in L}{\vee}\left(a \wedge\left(\tilde{E}_{(a)}(x) \wedge \tilde{E}_{(a)}(y)\right)\right) \\
& =\left(\underset{a \in L}{\vee}\left(a \wedge \tilde{E}_{(a)}(x)\right)\right) \wedge\left(\vee_{a \in L}\left(a \wedge \tilde{E}_{(a)}(y)\right)\right) \\
& =\mu(x) \wedge \mu(y) .
\end{aligned}
$$

Therefore $\tilde{E}$ is an $L$-fuzzy vector subspace.

We can define the operations between two $L$-fuzzy vector subspaces analogous to fuzzy vector subspaces.

Definition 3.7. Let $\tilde{E}_{1}=\left(E, \mu_{1}\right)$ and $\tilde{E}_{2}=\left(E, \mu_{2}\right)$ be two $L$-fuzzy vector subspaces on $E$. Define the intersection of $\tilde{E}_{1}$ and $\tilde{E}_{2}$ to be $\tilde{E}_{1} \cap \tilde{E}_{2}=\left(E, \mu_{1} \wedge \mu_{2}\right)$. Define the sum of $\tilde{E}_{1}$ and $\tilde{E}_{2}$ to be $\tilde{E}_{1}+\tilde{E}_{2}=\left(E, \mu_{1}+\mu_{2}\right)$ where $\mu_{1}+\mu_{2}$ is defined by for all $x \in E$

$$
\begin{aligned}
\left(\mu_{1}+\mu_{2}\right)(x) & =\underset{x=x_{1}+x_{2}}{\vee}\left(\mu_{1}\left(x_{1}\right) \wedge \mu_{2}\left(x_{2}\right)\right) \\
& =\underset{x_{1} \in E}{\vee}\left(\mu_{1}\left(x_{1}\right) \wedge \mu_{2}\left(x-x_{1}\right)\right) .
\end{aligned}
$$

Definition 3.8. Let $\tilde{E}_{1}=\left(E_{1}, \mu_{1}\right)$ and $\tilde{E}_{2}=\left(E_{2}, \mu_{2}\right)$ be two members of LFVS and $E=E_{1} \oplus E_{2}$. We define the direct sum of $\tilde{E}_{1}$ and $\tilde{E}_{2}$ to be $\tilde{E}_{1} \oplus \tilde{E}_{2}=\left(E, \mu_{1} \oplus \mu_{2}\right)$ where $\mu_{1} \oplus \mu_{2}$ is defined by for all $x \in E, x=x_{1} \oplus x_{2}, x_{i} \in E_{i}, i=1,2$

$$
\left(\mu_{1} \oplus \mu_{2}\right)(x)=\left(\mu_{1} \oplus \mu_{2}\right)\left(x_{1} \oplus x_{2}\right)=\mu_{1}\left(x_{1}\right) \wedge \mu_{2}\left(x_{2}\right) \text {. }
$$


Theorem 3.9. Let $\tilde{E}_{1}=\left(E, \mu_{1}\right)$ and $\tilde{E}_{2}=\left(E, \mu_{2}\right)$ be two members of LFVS on E. We have

(1) $\tilde{E}_{1} \cap \tilde{E}_{2}$ is a member of LFVS on $E$.

(2) $\tilde{E}_{1}+\tilde{E}_{2}$ is a member of LFVS on $E$.

The proof of the theorem is trivial and it is omitted.

Theorem 3.10. Let $\tilde{E}_{1}=\left(E, \mu_{1}\right)$ and $\tilde{E}_{2}=\left(E, \mu_{2}\right)$ be the members of LFVS. We have

(1) For all $a \in L,\left(\tilde{E}_{1} \cap \tilde{E}_{2}\right)_{[a]}=\left(\tilde{E}_{1}\right)_{[a]} \cap\left(\tilde{E}_{2}\right)_{[a]}$.

(2) For all $a \in L,\left(\tilde{E}_{1} \cap \tilde{E}_{2}\right)^{[a]}=\left(\tilde{E}_{1}\right)^{[a]} \cap\left(\tilde{E}_{2}\right)^{[a]}$.

(3) For any $a \in P(L),\left(\tilde{E}_{1} \cap \tilde{E}_{2}\right)^{(a)}=\left(\tilde{E}_{1}\right)^{(a)} \cap\left(\tilde{E}_{2}\right)^{(a)}$.

(4) For any $a \in P(L),\left(\tilde{E}_{1}+\tilde{E}_{2}\right)^{(a)}=\left(\tilde{E}_{1}\right)^{(a)}+\left(\tilde{E}_{2}\right)^{(a)}$.

Proof. The proofs of (1) and (2) are easy by the definition of $\tilde{E}_{1} \cap \tilde{E}_{2}$ and the properties of $L$-fuzzy sets.

(3) For any $a \in P(L)$, we have

$$
\begin{aligned}
x \in\left(\tilde{E}_{1} \cap \tilde{E}_{2}\right)^{(a)} & \Leftrightarrow \mu_{1}(x) \wedge \mu_{2}(x) \not \leq a \\
& \Leftrightarrow \mu_{1}(x) \not \leq a \text { and } \mu_{2}(x) \not \leq a \\
& \Leftrightarrow x \in\left(\tilde{E}_{1}\right)^{(a)} \cap\left(\tilde{E}_{2}\right)^{(a)}
\end{aligned}
$$

(4) By the definition of the sum of $L$-fuzzy vector subspaces, for any $a \in P(L)$ we have

$$
\begin{aligned}
x \in\left(\tilde{E}_{1}+\tilde{E}_{2}\right)^{(a)} & \Leftrightarrow \underset{x=x_{1}+x_{2}}{\vee}\left(\mu_{1}\left(x_{1}\right) \wedge \mu_{2}\left(x_{2}\right)\right) \not \leq a \\
& \Leftrightarrow \exists x_{1}, x_{2} \in E \text { and } x=x_{1}+x_{2}, \text { such that } \mu_{1}\left(x_{1}\right) \wedge \mu_{2}\left(x_{2}\right) \not \leq a . \\
& \Leftrightarrow \exists x_{1}, x_{2} \in E, \mu_{1}\left(x_{1}\right) \not \leq a \text { and } \mu_{2}\left(x_{2}\right) \not \leq a . \\
& \Leftrightarrow x=x_{1}+x_{2} \in \tilde{E}_{1}^{(a)}+\tilde{E}_{2}^{(a)} .
\end{aligned}
$$

Theorem 3.11. Let $\tilde{E}_{1}=\left(E, \mu_{1}\right)$ and $\tilde{E}_{2}=\left(E, \mu_{2}\right)$ be two members of LFVS. Suppose that for any $a, b \in L$, we have $\beta(a \wedge b)=\beta(a) \cap \beta(b)$. Then

(1) $\left(\tilde{E}_{1} \cap \tilde{E}_{2}\right)_{(a)}=\left(\tilde{E}_{1}\right)_{(a)} \cap\left(\tilde{E}_{2}\right)_{(a)}$,

(2) $\left(\tilde{E}_{1}+\tilde{E}_{2}\right)_{(a)}=\left(\tilde{E}_{1}\right)_{(a)}+\left(\tilde{E}_{2}\right)_{(a)}$.

The prove is trivial and omitted.

\section{Fuzzy Dimension of $L$-Fuzzy Vector Subspaces}

Definition 4.1. Let $\mathbb{N}(L)$ be the family of $L$-fuzzy natural number. The map $\operatorname{dim}:$ LFVS $\rightarrow \mathbb{N}(L)$ is defined by

$$
\operatorname{dim} \tilde{E}(n)=\underset{a \in L}{\vee}\left(a \wedge \operatorname{dim} \tilde{E}_{[a]}\right)(n)
$$

is called the $L$-fuzzy dimensional function of the $L$-fuzzy vector subspace $\tilde{E}$, and $\operatorname{dim} \tilde{E}$ is called the $L$-fuzzy dimension of $\tilde{E}$, it is an $L$-fuzzy natural number. We 
usually use another form of $\operatorname{dim} \tilde{E}$ as follows.

$$
\operatorname{dim} \tilde{E}(n)=\vee\left\{a \in L: \operatorname{dim} \tilde{E}_{[a]} \geq n\right\} .
$$

Theorem 4.2. For each $\tilde{E} \in \mathrm{LFVS}$ and $n \in \mathbb{N}$, we have

$$
\operatorname{dim} \tilde{E}(n)=\underset{a \in L}{\vee}\left(a \wedge \operatorname{dim} \tilde{E}_{(a)}\right)(n)=\vee\left\{a \in L: \operatorname{dim} \tilde{E}_{(a)} \geq n\right\}
$$

Proof. For any $n \in \mathbb{N}$, let $\lambda=\underset{a \in L}{\vee}\left(a \wedge \operatorname{dim} \tilde{E}_{(a)}\right)(n)$. Obviously $\lambda \leq \operatorname{dim} \tilde{E}(n)$. Next we show that $\lambda \geq \operatorname{dim} \tilde{E}(n)$. Suppose that $b \in L$ and $b \in \beta(\operatorname{dim} \tilde{E}(n))$, then there exists $a \in L$ and $\operatorname{dim} \tilde{E}_{[a]} \geq n$ such that $b \in \beta(a)$. In this case, $n \leq \operatorname{dim} \tilde{E}_{[a]} \leq \operatorname{dim} \tilde{E}_{(b)} \leq \operatorname{dim} \tilde{E}_{[b]}$ which implies $\lambda=\vee\left\{a \in L: \operatorname{dim} \tilde{E}_{(a)} \geq n\right\} \geq b$. Thus we have

$$
\lambda \geq \vee\{b \mid b \in \beta(\operatorname{dim} \tilde{E}(n))\}=\operatorname{dim} \tilde{E}(n) .
$$

This completes the proof.

Theorem 4.3. Let the pair $\tilde{E}=(E, \mu)$ be a member of LFVS. Then for any $a \in L$,

$$
(\operatorname{dim} \tilde{E})_{(a)} \leq \operatorname{dim} \tilde{E}_{[a]} \leq(\operatorname{dim} \tilde{E})_{[a]} .
$$

If $\beta(a \wedge b)=\beta(a) \cap \beta(b)$ for all $a, b \in L$, then

$$
(\operatorname{dim} \tilde{E})_{(a)} \leq \operatorname{dim} \tilde{E}_{(a)} \leq \operatorname{dim} \tilde{E}_{[a]} \leq(\operatorname{dim} \tilde{E})_{[a]} .
$$

In particular, $(\operatorname{dim} \tilde{E})_{[a]}=\operatorname{dim} \tilde{E}_{[a]}$ for any $a \in J(L)$.

Proof. In order to prove $(\operatorname{dim} \tilde{E})_{(a)} \leq \operatorname{dim} \tilde{E}_{(a)}$. Suppose that $n \leq(\operatorname{dim} \tilde{E})_{(a)}$, then $a \in \beta(\operatorname{dim} \tilde{E}(n))$. Since $\beta$ is a preserve-union map, there is $b \in L$ and $n \leq \operatorname{dim} \tilde{E}_{[b]}$, such that $a \in \beta(b)$. Because $\tilde{E}_{[b]} \subseteq \tilde{E}_{(a)} \subseteq \tilde{E}_{[a]}$, thus $n \leq \operatorname{dim} \tilde{E}_{(a)}$. Therefore $(\operatorname{dim} \tilde{E})_{(a)} \leq \operatorname{dim} \tilde{E}_{(a)}$.

$\operatorname{dim} \tilde{E}_{(a)} \leq \operatorname{dim} \tilde{E}_{[a]}$ is obvious. Moreover, we can obtain that $\operatorname{dim} \tilde{E}_{[a]} \leq(\operatorname{dim} \tilde{E})_{[a]}$ from the definition of $\operatorname{dim}(\tilde{E})$.

In order to prove for any $a \in J(L),(\operatorname{dim} \tilde{E})_{[a]}=\operatorname{dim} \tilde{E}_{[a]}$, we only need to show $(\operatorname{dim} \tilde{E})_{[a]} \subseteq \operatorname{dim} \tilde{E}_{[a]}$. Since the set $\mu(E)$ is finite, for any $a \in J(L)$ we have

$$
\begin{aligned}
n \leq(\operatorname{dim} \tilde{E})_{[a]} & \Rightarrow \operatorname{dim} \tilde{E}(n) \geq a \\
& \Rightarrow \vee\left\{b \in L: \operatorname{dim} \tilde{E}_{[b]} \geq n\right\} \geq a \\
& \Rightarrow \exists a \leq b, \text { such that } n \leq \operatorname{dim} \tilde{E}_{[b]} \\
& \Rightarrow n \leq \operatorname{dim} \tilde{E}_{[a]}
\end{aligned}
$$

Therefore $(\operatorname{dim} \tilde{E})_{[a]}=\operatorname{dim} \tilde{E}_{[a]}$. 
Theorem 4.4. Let $\tilde{E}=(E, \mu)$ be a member of LFVS. Then

$$
(\operatorname{dim} \tilde{E})^{(a)} \leq \operatorname{dim} \tilde{E}^{(a)} \leq \operatorname{dim} \tilde{E}^{[a]} \leq(\operatorname{dim} \tilde{E})^{[a]} .
$$

In particular, $(\operatorname{dim} \tilde{E})^{(a)}=\operatorname{dim} \tilde{E}^{(a)}$ for any $a \in P(L)$.

Proof. $(\operatorname{dim} \tilde{E})^{(a)} \leq \operatorname{dim} \tilde{E}^{(a)}$ can be proved from the following implications.

$$
\begin{aligned}
n \leq(\operatorname{dim} \tilde{E})^{(a)} & \Leftrightarrow \operatorname{dim} \tilde{E}(n) \not \leq a \\
& \Leftrightarrow \vee\left\{b \in L: \operatorname{dim} \tilde{E}_{[b]} \geq n\right\} \not \leq a \\
& \Leftrightarrow \exists b \not \leq a \text {, such that } n \leq \operatorname{dim} \tilde{E}_{[b]} \\
& \Rightarrow \operatorname{dim} \tilde{E}^{(a)}=\operatorname{dim}\left(\bigcup_{b \nsubseteq a} \tilde{E}_{[b]}\right) \geq n .
\end{aligned}
$$

Let $a \in P(L)$. In order to show $\operatorname{dim} \tilde{E}^{(a)} \leq(\operatorname{dim} \tilde{E})^{(a)}$, we need to show that $\operatorname{dim}\left(\bigcup_{b \nsubseteq a} \tilde{E}_{[b]}\right) \leq \underset{b \nless a}{\vee} \operatorname{dim} \tilde{E}_{[b]}$. Suppose that $n \leq \operatorname{dim}\left(\bigcup_{b \nsubseteq a} \tilde{E}_{[b]}\right)$. Since the number of $\tilde{E}_{[a]}$ is finite, then when $b \not \subset a$, the number of $\tilde{E}_{[b]}$ is finite, denotes $\tilde{E}_{\left[a_{1}\right]}, \tilde{E}_{\left[a_{2}\right]}, \cdots, \tilde{E}_{\left[a_{r}\right]}$, where $a_{i} \not \leq a$ for any $i \in\{1,2, \cdots, r\}$. Thus $\bigcup_{b \nsubseteq a} \tilde{E}_{[b]}=\bigcup_{i=1}^{r} \tilde{E}_{\left[a_{i}\right]}$. Since $a \in P(L)$, then we have $c=a_{1} \wedge a_{2} \wedge \cdots \wedge a_{r} \not \leq a$. Further we have $\bigcup_{i=1}^{r} \tilde{E}_{\left[a_{i}\right]} \subseteq \tilde{E}_{[c]}$. Thus for any

$$
n \leq \operatorname{dim}\left(\bigcup_{b \nless a} \tilde{E}_{[b]}\right)=\operatorname{dim}\left(\bigcup_{i=1}^{r} \tilde{E}_{\left[a_{i}\right]}\right) \leq \operatorname{dim} \tilde{E}_{[c]} \leq \underset{b \nless a}{\vee} \operatorname{dim} \tilde{E}_{[b]} \leq \underset{b \nless a}{\vee}(\operatorname{dim} \tilde{E})_{[b]}=(\operatorname{dim} \tilde{E})^{(a)} .
$$

Therefore for any $a \in P(L),(\operatorname{dim} \tilde{E})^{(a)}=\operatorname{dim} \tilde{E}^{(a)}$.

$\operatorname{dim} \tilde{E}^{(a)} \leq \operatorname{dim} \tilde{E}^{[a]}$ is obvious. We show that $\left.\operatorname{dim} \tilde{E}^{[a]} \leq(\operatorname{dim} \tilde{E})\right)^{[a]}$ in the following implications.

$$
\begin{aligned}
\operatorname{dim} \tilde{E}^{[a]} & =\operatorname{dim} \bigcap_{\substack{a \in \alpha(b) \\
b \in P(L)}} \tilde{E}^{(b)} \leq \underset{\substack{a \in \alpha(b) \\
b \in P(L)}}{\wedge} \operatorname{dim} \tilde{E}^{(b)} \\
& =\underset{\substack{a \in \alpha(b) \\
b \in P(L)}}{\wedge}(\operatorname{dim} \tilde{E})^{(b)}=(\operatorname{dim} \tilde{E})^{[a]} .
\end{aligned}
$$

Theorem 4.5. Let $\tilde{E}_{1}=\left(E, \mu_{1}\right)$ and $\tilde{E}_{2}=\left(E, \mu_{2}\right)$ be two L-fuzzy vector subspaces. Then the following equality holds

$$
\operatorname{dim}\left(\tilde{E}_{1}+\tilde{E}_{2}\right)+\operatorname{dim}\left(\tilde{E}_{1} \cap \tilde{E}_{2}\right)=\operatorname{dim} \tilde{E}_{1}+\operatorname{dim} \tilde{E}_{2} .
$$

Proof. We denote the sum of $\tilde{E}_{1}$ and $\tilde{E}_{2}$ by $\tilde{E}_{1}+\tilde{E}_{2}=(E, \mu)$. From Theorem 11, we know that $\tilde{E}_{1}+\tilde{E}_{2}$ is a $L$-fuzzy vector subspace. By the properties of $L$-fuzzy natural numbers, Theorem 12 and the dimensional formulation of vector spaces, we know for any $a \in P(L)$, 


$$
\begin{aligned}
& \left(\operatorname{dim}\left(\tilde{E}_{1}+\tilde{E}_{2}\right)+\operatorname{dim}\left(\tilde{E}_{1} \cap \tilde{E}_{2}\right)\right)^{(a)} \\
= & \left(\operatorname{dim}\left(\tilde{E}_{1}+\tilde{E}_{2}\right)\right)^{(a)}+\left(\operatorname{dim}\left(\tilde{E}_{1} \cap \tilde{E}_{2}\right)\right)^{(a)} \\
= & \operatorname{dim}\left(\tilde{E}_{1}+\tilde{E}_{2}\right)^{(a)}+\operatorname{dim}\left(\tilde{E}_{1} \cap \tilde{E}_{2}\right)^{(a)} \\
= & \operatorname{dim}\left(\tilde{E}_{1}^{(a)}+\tilde{E}_{2}^{(a)}\right)+\operatorname{dim}\left(\tilde{E}_{1}^{(a)} \cap \tilde{E}_{2}^{(a)}\right) \\
= & \operatorname{dim} \tilde{E}_{1}^{(a)}+\operatorname{dim} \tilde{E}_{2}^{(a)}-\operatorname{dim}\left(\tilde{E}_{1}^{(a)} \cap \tilde{E}_{2}^{(a)}\right)+\left(\operatorname{dim}\left(\tilde{E}_{1}^{(a)} \cap \tilde{E}_{2}^{(a)}\right)\right. \\
= & \operatorname{dim} \tilde{E}_{1}^{(a)}+\operatorname{dim} \tilde{E}_{2}^{(a)}
\end{aligned}
$$

Therefore $\operatorname{dim}\left(\tilde{E}_{1}+\tilde{E}_{2}\right)+\operatorname{dim}\left(\tilde{E}_{1} \cap \tilde{E}_{2}\right)=\operatorname{dim} \tilde{E}_{1}+\operatorname{dim} \tilde{E}_{2}$.

Definition 4.6. Suppose that $\tilde{E}=(E, \mu)$ is an $L$-fuzzy vector subspace. A map $f: E \rightarrow E$ is called an $L$-fuzzy linear transformation, if it satisfies the following conditions:

(1) $f$ is a linear map on $E$.

(2) For all $x \in E, \mu(f(x)) \geq \mu(x)$.

Theorem 4.7. Suppose that $\tilde{E}=(E, \mu)$ is an L-fuzzy vector subspace, $f$ is an $L$-fuzzy linear transformation on $E$, then $\widetilde{\operatorname{ker}} f=\left(\operatorname{ker} f,\left.\mu\right|_{\mathrm{ker}}\right)$ and $\widetilde{\operatorname{im}} f=\left(\operatorname{im} f,\left.\mu\right|_{\text {imf }}\right)$ are L-fuzzy vector subspaces.

The prove is trivial and omitted.

Theorem 4.8. Suppose that $\tilde{E}=(E, \mu)$ is an L-fuzzy vector subspace, $f: E \rightarrow E$ is an L-fuzzy linear transformation, then

$$
\operatorname{dim}(\widetilde{\operatorname{ker}} f)+\operatorname{dim}(\widetilde{\operatorname{im}} f)=\operatorname{dim} \tilde{E}
$$

Proof. Suppose that $\varphi$ is a linear transformation on (crisp) vector spaces $V$, then the equality $\operatorname{dim}(\operatorname{im} \varphi)+\operatorname{dim}(\operatorname{kef} \varphi)=\operatorname{dim} V$ holds. Hence, for all $a \in P(L)$, we have

$$
\begin{aligned}
(\operatorname{dim}(\widetilde{\operatorname{im}} f)+\operatorname{dim}(\widetilde{\operatorname{ker} f}))^{(a)} & =(\operatorname{dim}(\widetilde{\operatorname{im}} f))^{(a)}+(\operatorname{dim}(\widetilde{\operatorname{ker}} f))^{(a)} \\
& =\operatorname{dim}(\widetilde{\operatorname{im}} f)^{(a)}+\operatorname{dim}(\widetilde{\operatorname{ker} f})^{(a)} \\
& =\operatorname{dim}\left(\tilde{E}^{(a)} \cap \operatorname{im} f\right)+\operatorname{dim}\left(\tilde{E}^{(a)} \cap \operatorname{kef} f\right)
\end{aligned}
$$

Since $\left.f\right|_{\tilde{E}^{(a)}}$ is a linear transformation on $\tilde{E}^{(a)}$, we have

$$
\begin{aligned}
(\operatorname{dim}(\widetilde{\operatorname{im} f})+\operatorname{dim}(\widetilde{\operatorname{ker} f}))^{(a)} & =\operatorname{dim}\left(\left.\operatorname{im} f\right|_{\tilde{E}^{(a)}}\right)+\operatorname{dim}\left(\left.\operatorname{kef} f\right|_{\tilde{E}^{(a)}}\right) \\
& =\operatorname{dim} \tilde{E}^{(a)}=(\operatorname{dim} \tilde{E})^{(a)} .
\end{aligned}
$$

Therefore $\operatorname{dim}(\widetilde{\operatorname{ker} f})+\operatorname{dim}(\widetilde{\operatorname{im} f})=\operatorname{dim} \tilde{E}$.

\section{Conclusion}

In this paper, $L$-fuzzy vector subspace is defined and showed that its dimension is an $L$-fuzzy natural number. Based on the definitions, some good properties of crisp vector spaces are hold in a finite-dimensional $L$-fuzzy vector subspace. In particular, the equality $\operatorname{dim}\left(\tilde{E}_{1}+\tilde{E}_{2}\right)+\operatorname{dim}\left(\tilde{E}_{1} \cap \tilde{E}_{2}\right)=\operatorname{dim} \tilde{E}_{1}+\operatorname{dim} \tilde{E}_{2}$ holds without any restricted 
conditions. At the same time, $\operatorname{dim}(\widetilde{\operatorname{im}} f)+\operatorname{dim}(\widetilde{\operatorname{kef}} f)=\operatorname{dim} \tilde{E}$ holds.

\section{Acknowledgements}

The authors would like to thank the reviewers for their valuable comments and suggestions.

\section{Fund}

The project is by the Science \& Technology Program of Beijing Municipal Commission of Education (KM201611417007), the NNSF of China (11371002), the academic youth backbone project of Heilongjiang Education Department (1251G3036), the foundation of Heilongjiang Province (A201209).

\section{References}

[1] Katsaras, A.K. and Liu, D.B. (1977) Fuzzy Vector Spaces and Fuzzy Topological Vector Spaces. Journal of Mathematical Analysis and Applications, 58, 135-146. https://doi.org/10.1016/0022-247X(77)90233-5

[2] Lubczonok, G. and Murali, V. (2002) On Flags and Fuzzy Subspaces of Vector Spaces. Fuzzy Sets and Systems, 125, 201-207. https://doi.org/10.1016/S0165-0114(00)00129-9

[3] Abdukhalikov, K.S., Tulenbaev, M.S. and Umirbarv, U.U. (1994) On Fuzzy Bases of Vector Spaces. Fuzzy Sets and Systems, 63, 201-206. https://doi.org/10.1016/0165-0114(94)90350-6

[4] Abdukhalikov, K.S. (1996) The Dual of a Fuzzy Subspace. Fuzzy Sets and Systems, 82, 375381. https://doi.org/10.1016/0165-0114(96)84977-3

[5] Lubczonok, P. (1990) Fuzzy Vector Spaces. Fuzzy Sets and Systems, 38, 329-343. https://doi.org/10.1016/0165-0114(90)90206-L

[6] Lowen, R. (1980) Convex Fuzzy Sets. Fuzzy Sets and Systems, 3, 291-310. https://doi.org/10.1016/0165-0114(80)90025-1

[7] Shi, F.G. and Huang, C.E. (2010) Fuzzy Bases and the Fuzzy Dimension of Fuzzy Vector Spaces. Mathematical Communications, 15, 303-310.

[8] Gierz, G., et al. (2003) Continuous Lattices and Domains. Encyclopedia of Mathematics and its Applications, Cambridge University Press, Cambridge. https://doi.org/10.1017/CBO9780511542725

[9] Dwinger, P. (1982) Characterizations of the Complete Homomorphic Images of a Completely Distributive Complete Lattice I. Indagationes Mathematicae (Proceedings), 85, 403414. https://doi.org/10.1016/1385-7258(82)90034-8

[10] Wang, G.-J. (1992) Theory of Topological Molecular Lattices. Fuzzy Sets and Systems, 47, 351-376. https://doi.org/10.1016/0165-0114(92)90301-J

[11] Huang, H.-L. and Shi, F.-G. (2008) L-Fuzzy Numbers and Their Properties. Information Sciences, 178, 1141-1151. https://doi.org/10.1016/j.ins.2007.10.001

[12] Shi, F.-G. (2000) L-Fuzzy Relation and L-Fuzzy Subgroup. Journal of Fuzzy Mathematics, 8, 491-499.

[13] Negoita, C.V. and Ralescu, D.A. (1975) Applications of Fuzzy Sets to Systems Analysis, Interdisciplinary Systems Research Series 11, Birkhaeuser, Basel. https://doi.org/10.1007/978-3-0348-5921-9

[14] Shi, F.-G. (1995) Theory of $L_{\beta}$-Nested Sets and $L_{\alpha}$-Nested Sets and Its Applications. Fuzzy 
Systems and Mathematics, 4, 65-72. (In Chinese)

[15] Shi, F.-G. (1996) L-Fuzzy Sets and Prime Element Nested Sets. Journal of Mathematical Research and Exposition, 16, 398-402. (In Chinese)

[16] Shi, F.-G. (1996) Theory of Molecular Nested Sets and Its Applications. Journal of Yantai Teachers University, 1, 33-36. (In Chinese)

[17] Shi, F.-G. (2009) A New Approach to the Fuzzification of Matroids. Fuzzy Sets and Systems, 160, 696-705. https://doi.org/10.1016/j.fss.2008.05.007

\section{Submit or recommend next manuscript to SCIRP and we will provide best service} for you:

Accepting pre-submission inquiries through Email, Facebook, LinkedIn, Twitter, etc. A wide selection of journals (inclusive of 9 subjects, more than 200 journals)

Providing 24-hour high-quality service

User-friendly online submission system

Fair and swift peer-review system

Efficient typesetting and proofreading procedure

Display of the result of downloads and visits, as well as the number of cited articles

Maximum dissemination of your research work

Submit your manuscript at: http://papersubmission.scirp.org/

Or contact alamt@scirp.org 